\section{Vagaries of nomenclature}

SIR-It is a pity that when zoological nomenclature is an Aunt Sally for nontaxonomists they should so often be uninformed or disingenuous. Seldom do they trouble to find a reason for a nomenclature requirement that seems to them arcane or futile. So it is with L.J. BruceChwatt's remonstrance (Nature 325, 114; 1987) against the use of '- $i$ ' and '-ii' terminations for patronymic names of mosquito species. He argues that the '-ii' ending is 'wrong', because names such as bancroftii and cruzii honour Bancroft and Cruz, not Bancroftius and Cruzius, and therefore that by his lopping of the terminal ' $-i$ ' when there are two he is using the 'grammatically correct spelling'.

This would be fine were it not for the fact that notional latinization of modern personal names (to Bancroftius, for example) before forming the genitives has been widespread and legitimate practice since the dawn of Latin-based nomenclature and is still permissible today. Would he have us 'correct' the multitude of such '-ii' names published since 1758 in every major animal group — or is he really after a dispensation for malariologists? Anyway, he should take heart. The use of '-ii' personal genitives for new species has been effectively dead for many years and is recommended against in the International Code of Zoological Nomenclature (Appendix D(III)16(b)).

Bruce-Chwatt fails to note the obvious risk inherent in what he has done, namely that those without his classical grounding (most of us), seeing that he reduces '- $i i$ ' to ' $-i$ ', might assume that the second ' $i$ ' should always be lopped. He does not mention the point, but the names of several mosquitoes correctly end '-ii' because they are genitives based on personal names (usually Italian, Indian or Japanese) that happen to end with the letter ' $i$ '; ficalbii, forattinii, goeldii, hatorii, martinii, onorii, purii, are examples. There is also fabricii from the great (latinized) naturalist Fabricius and the geographically derived mississippii.

Under the present code, the correct spelling is the one published when the species was first described, whether or not the ' $-i$ ' or '-ii' termination was used. This allows the correct spelling to be objectively determined by reference to that description - and obviates the need to trace all the subsequent history of the name in its scattered literature in an effort to discover who first altered it (or indeed whether it has ever been altered at all). Under the code all is clear; a variant subsequent spelling (one ' $i$ ' reduced to two or vice versa) is an 'incorrect subsequent spelling' (Article 33(d)) and cannot be validly used. The much-maligned and misunderstood code is right on this particular issue.
I cannot refrain from noting the paradox with which Bruce-Chwatt ends his letter. He blames the code for preserving stability, an unsettling experience for taxonomists used to obloquy for supposedly doing the opposite.

R.W. CROSSKEY

Department of Entomology,

British Museum (Natural History), Cromwell Road, London SW7 5BD, UK

\section{Divergence and directional mutation pressures}

SIR-Grivell' proposes that divergent genetic codes, as found in ciliated protozoa and Mycoplasma may be "relics of a primitive genetic code [that] possibly preceded the emergence of the so-called universal code". We suggest that a different explanation is more probable, and that such codes may be formed by capture of stop codons. Clues to this event in the case of ciliates are found by comparing the sequences of transfer RNAs.

Kuchino, Hanyu and co-workers ${ }^{2,3}$ found two 'new' glutamine tRNAs in Tetrahymena thermophilus, one with the anticodon UmUA (where Um is $2^{\prime}-O$ - methyluridine), pairing with UAA and UAG, and the other with anticodon CUA, pairing with UAG. UAA and UAG are stop codons in the universal code, but in ciliated protozoa the only stop codon appears to be UGA. Differences in nucleotide sequences between the 'new' tRNAs and the other glutamine tRNA, with anticodon UmUG, in Tetrahymena, are 13 and 11 nucleotide substitutions in tRNA $_{U m U G}^{\mathrm{Gln}}$ when compared with tRNA $\mathrm{U}_{\mathrm{UmUA}}^{\mathrm{Gln}}$ and tRNA ${ }_{\text {CUA }}^{\text {Gin }}$ respectively, and 4 substitutions in $\mathrm{TRNA}_{\mathrm{UmUA}}^{\mathrm{Gln}}$ compared with $\operatorname{tRNA}_{\mathrm{CUA}}^{\mathrm{Gin}}$ (ref. 3). These differences may be a rough clue as to time elapsed since evolutionary divergence 4 . Differences between yeast and rat or wheat tRNAs with the same anticodon are 13-15 substitutions $s^{5}$ and between Drosophila and vertebrates are $5-12$ substitutions ${ }^{5}$. From this, one could conclude that the divergences of the tRNAs for Gln in Tetrahymena were well within the time of existence of eukaryotes.

Capture of stop codons could have taken place through a series of nondeleterious (neutral or adaptive) changes brought about by 'directional mutational pressure". Stop codons UAA and UAG have evidently disappeared from ciliates, perhaps during successive episodes of AT and GC pressure. AT pressure presumably now exists in ciliates, as shown by the low GC content (20-30 per cent) of their DNA. The gene for $\mathrm{tRNA}_{\mathrm{UmUG}}^{\mathrm{Gln}}$ duplicated and one of the duplicates, under AT pressure, acquired anticodon UmUA. Later, the tRNA gene with anticodon UmUA duplicated again, and in one of the duplicates, anticodon UmUA mutated to
CUA, which pairs strongly with $\mathrm{UAG}^{3}$.

During this period, some of the glutamine codons CAA and CAG mutated under AT pressure to UAA and UAG, pairing with the new glutamine anticodons UmUA and CUA. UAA and UAG have been identified as alternate glutamine codons in the ciliated protozoans Tetrahymena $^{7}$, Stylonichia ${ }^{8}$ and Paramecium ${ }^{9,10}$. This may be a result of recent stop codon capture $^{11}$. This is in contrast to the suggestion by Grivell' that "the ciliates branched off from the primitive eukaryotic ancestor very early in evolution, quite possibly at a time when protein translation was still tolerant of changes in the genetic code". No such tolerances need be postulated, for the observed changes are compatible with neutral substitutions, and account for the presence in Tetrahymena, of four glutamine codons, CAA, CAG, UAA and UAG, and three glutamine anticodons, UUG, UUA and CUA. An analogous pattern exists for leucine in the universal genetic code with codons CUA, CUG, UUA and UUG and anticodons, UAG, UAA and CAA.

Similarly, in Mycoplasma capricolum (25 per cent GC in DNA) AT pressure has resuited in the disappearance of UGA stop codons, replaced by UAA, and the mutation of anticodon CCA, in a duplicate of tryptophan tRNA, to $\mathrm{UCA}^{12}$. Simultaneously, tryptophan codons UGG mutated to UGA. Anticodon UCA pairs with both UGG and UGA by 'wobble'. UGA has become a tryptophan codon, 'captured' by AT pressure ${ }^{12,13}$.

We propose that Tetrahymena and Mycoplasma have both evolved to make translatable use of former stop codons ${ }^{14}$ and that the divergent code in ciliates may be of recent origin and derived from the eukaryotic code.

University of California, Berkeley,

6701 San Pablo Avenue,

Oakland, California 94608, USA

Syozo Osawa Akira Muto

Laboratory of Molecular Genetics,

Nagoya University,

Nagoya 464, Japan

1. Grivell, L.A. Nature 324, 109-110 (1986).

2. Kuchino, Y., Hanyu, N., Tashiro, F. \& Nishimura, S. Proc. natn. Acad. Sci. U.S.A. 82, 4758-4762 (1985).

3. Hanyu, N., Kuchino, Y. \& Nishimura, S. EMBO J. 5 , 1307-1311 (1986).

4. Holmquist, R., Jukes, T.H. \& Pangburn, S. J. molec. Biol. 78, 91-116 (1973)

5. Sprinzl, M., Vorderwiibecke, T. \& Hartmann, T. Nucleic Acid Res. 13, r51-r104 (1985).

6. Sueoka, N. Proc. natn. Acad. Sci. U.S.A 48, $582-592$ (1962)

7. Horowitz, S. \& Gorovsky, M.A. Proc. natn. Acad. Sci. U.S.A. 82, 2452-2455 (1985).

8. Helftenbein, E. Nucleic Acids Res. 13, 415-433 (1985)

9. Preer, J.R. Jr, Preer, L.B., Rudman, B.M. \& Barnett, A.J. Nature 314, 188-190 (1985)

10. Caron, F. \& Meyer, E. Nature 314, 185-188 (1985)

11. Lehman, N. thesis, Univ. California (1986).

12. Yamao, F. et al. Proc. natn. Acad. Sci. U.S.A. 82, 23062309 (1985).

13. Jukes, T.H. J. molec. Evol. 22, 361-362 (1985) 